\title{
Time-resolved SANS visualization of hierarchical structures during thermochemical reactions
}

\author{
Sai Venkatesh Pingali ${ }^{1}$ (pingalis@ornl.gov), Riddhi Shah, ${ }^{1,2}$ Shawn Mansfield, ${ }^{3}$ Volker Urban, ${ }^{1}$ \\ Paul Langan, ${ }^{1}$ Brian H. Davison, ${ }^{1,2}$ and Hugh O’Neill ${ }^{1,2}$. \\ ${ }^{1}$ Oak Ridge National Laboratory, Oak Ridge, Tennessee \\ ${ }^{2}$ Bredesen Center, University of Tennessee, Knoxville, Tennessee \\ ${ }^{3}$ University of British Columbia, Vancouver, Canada
}

Knowledge of structural changes to biomass during pretreatment will enable an informed approach to improving biomass conversion efficiencies. We developed a pressure reaction cell to monitor morphological changes in biomass using small-angle neutron scattering (SANS) during thermochemical pretreatment in real-time. This approach takes advantage of the non-destructive and high penetration properties of neutrons to perform in-situ studies. Recently, we have extended the capabilities of the Bio-SANS instrument at the High Flux Isotope Reactor at Oak Ridge National Laboratory by installation of an additional detector array. The upgrade makes it possible to capture neutrons scattered at higher angles simultaneously to the neutrons captured at smaller scattering angles by the main detector. As a result, data collection times are dramatically reduced and it is possible to obtain structural information at much shorter length scales than was previously possible. Here, we report on real-time SANS studies of native poplar and variant that is deficient in lignin synthesis, during dilute acid and alkali pretreatments. We observe significant differences in lignin aggregation patterns in the native and mutant poplar comparing the two pretreatment regimes. On the other hand, the scattering signature assigned to the cellulose microfibrils remains relatively unchanged in both pretreatments. A quantitative analysis of the structural changes in the native and mutant plants will be presented. Using this approach, it is possible to obtain molecular level insights into structural rearrangements of biomass polymers during pretreatment to better understand the consequences of genetic mutations on the overall digestability of biomass.

http://www.ornl.gov/science-discovery/clean-energy/research-areas/systems-biology/bioenergy/dynamicvisualization-of-lignocellulose 